\title{
ADIABATIC SHEARING FLOW CAUSED BY TIME DEPENDENT INERTIAL FORCE*
}

\author{
BY \\ NICOLAS CHARALAMBAKIS \\ Brown University and University of Thessaloniki, Greece
}

\begin{abstract}
We consider the shearing adiabatic flow of an incompressible Neutonian fluid with temperature dependent viscosity caused by a time dependent "oscillatory" body force. We show that, as $t \rightarrow \infty$, the flow converges with exponential rate to a rigid "oscillatory" motion at fixed temperature.
\end{abstract}

1. Introduction. We consider an incompressible Newtonian fluid with temperature dependent viscosity in an adiabatic shearing flow caused by a time dependent body force $f(t)$. The fluid is confined in the strip between the planes $x=0$ and $x=1$. The contact of the fluid with these planes is assumed frictionless. The body force $f(t)$ and thereby the flow is in the direction of the axis $y$, perpendicular to $x$.

We assume the fluid has unit density and unit specific heat, so its internal energy is identical to temperature, i.e., $e=\theta$. On the other hand the stress is given by

$$
\sigma(x, t)=\mu(\theta(x, t)) v_{x}(x, t),
$$

where the viscosity $\mu(\theta)$ is a know, $C^{2}$ smooth, function which satisfies

$$
\begin{aligned}
\mu(\theta) & >0, \\
\mu^{\prime}(\theta) & \leqslant 0, \\
\int_{0}^{\infty} \mu(\theta) d \theta & =\infty .
\end{aligned}
$$

Thus, the balance laws of momentum and energy take the form

$$
\begin{aligned}
& v_{t}(x, t)=\sigma_{x}(x, t)+f(t) \\
& \theta_{t}(x, t)=\sigma(x, t) v_{x}(x, t) .
\end{aligned}
$$

Since the contact of the fluid with the boundary is frictionless,

$$
\sigma(1, t)=\sigma(0, t)=0, \quad 0 \leqslant t<\infty .
$$

We specify initial conditions

$$
v(x, 0)=v_{0}(x), \quad \theta(x, 0)=\theta_{0}(x), \quad 0 \leqslant x \leqslant 1,
$$

*Received March 25, 1983. This research has been supported in part by the United States Army Research Office under contract \# DAAG-29-79-C-0161. 
with

$$
v_{x}(0,0)=v_{x}(0,0)=v_{x}(1,0)=0 .
$$

We assume the body force is "oscillatory", in the sense

$$
\left|\int_{0}^{t} f(\tau) d \tau\right| \leqslant K<\infty, \quad 0 \leqslant t<\infty .
$$

Then, (1.1), (1.5), (1.6) and (1.7) admit the solution

$$
\begin{aligned}
& \bar{v}(x, t)=v_{1}+\int_{0}^{t} f(\tau) d \tau, \\
& \bar{\theta}(x, t)=\theta_{1}(x),
\end{aligned}
$$

which represents a rigid motion at constant (in time) temperature.

Our objective is to investigate whether this rigid motion is asymptotically stable, i.e., whether the solution $(v(x, t), \theta(x, t))$ of $(1.1),(1.5),(1.6),(1.7),(1.8)$ has, as $t \rightarrow \infty$, the asymptotic form (1.11), (1.12), independently of $v(x), \theta_{0}(x)$.

We will show the following

Theorem. Assume $v_{0}(x) \in W^{2,2}(0,1), \theta_{0}(x) \in W^{1,2}(0,1), \theta_{0}(x)>0,0 \leqslant x \leqslant 1$.

Then there exists a unique classical solution of (1.1), (1.5), (1.6), (1.7), (1.8) and (1.9) on $[0,1] \times[0, \infty)$ and, as $t \rightarrow \infty$,

$$
\begin{aligned}
& v(x, t)=\int_{0}^{t} f(\tau) d \tau+\int_{0}^{1} v_{0}(x) d x+O\left(e^{-K t}\right), \\
& \sigma(x, t)=O\left(e^{-K t}\right), \\
& \theta(x, t) \rightarrow \theta_{1}(x)>0 \\
&\left|\theta_{x}(x, t)\right| \leqslant K,
\end{aligned}
$$

uniformly in $x$ on $[0,1]$.

The proof of this theorem, presented in Section 2, is based on a priori estimates for solutions of (1.1), (1.5), (1.6), (1.7) and (1.8) under the assumed properties of the functions $f(t)$ and $\mu(\theta)$. Essentially we show that viscosity in the momentum balance equation (1.5) wins over the destabilizing effect of stress power in the energy balance equation (1.6) and enforces the decay of the velocity gradient to zero.

The problem of uniform shearing of a fluid of this type was investigated in [1] and an analogous asymptotic stability result was established there.

Acknowledgement. I thank Professor Constantine Dafermos for several discussions during the preparation of this work. The paper was written while I was a visitor in the Lefschetz Center for Dynamical Systems, Division of Applied Mathematics, Brown University, Providence, R. I.

2. Proof of the theorem. In this section we assume that $(v(x, t), \theta(x, t))$ is a solution of (1.1), (1.5), (1.6), (1.7), (1.8) on $[0,1] \times[0, \infty)$ such that $v(\cdot, t), v_{x}(\cdot, t), v_{t}(\cdot, t), v_{x x}(\cdot, t)$, $\theta(\cdot, t), \theta_{x}(\cdot, t)$ are all in $C^{0}\left([0, \infty) ; L^{2}(0,1)\right), v_{x t}(\cdot, t)$ is in $C^{0}\left((0, \infty) ; L^{2}(0,1)\right)$ and $v_{t t}(\cdot, t)$ is in $L_{\mathrm{loc}}^{2}\left((0, \infty) ; L^{2}(0,1)\right)$. 
Throughout this section, $K$ will denote a generic constant which can be estimated in terms of properties of the viscosity function $\mu(\theta)$ and upper bounds of the $W^{2,2}(0,1)$ norm of $v_{0}(x)$ and the $W^{1,2}(0,1)$ norm of $\theta_{0}(x)$.

LEMMA 2.1 .

$$
\begin{array}{r}
\int_{0}^{t} \int_{0}^{1} \sigma_{x}^{2}(x, \tau) d x d \tau \leqslant K, \\
\int_{0}^{t} \sigma^{2}(x, \tau) d \tau \leqslant K, \\
\int_{0}^{1} \sigma^{2}(x, \tau) d x \leqslant K .
\end{array}
$$

Proof. We multiply $(1.5)$ by $\left(v_{t}(x, t)-f(t)\right)$, we integrate over $[0,1] \times[0, t]$ and integrate by parts to obtain

$$
\int_{0}^{t} \int_{0}^{1}\left[v_{t}(x, \tau)-f(\tau)\right]^{2} d x d \tau+\int_{0}^{t} \int_{0}^{1} v_{t x}(x, \tau) \sigma(x, \tau) d x d \tau=0
$$

Then, using (1.1),

$$
\int_{0}^{t} \int_{0}^{1}\left[v_{t}(x, \tau)-f(\tau)\right]^{2} d x d \tau+\int_{0}^{t} \int_{0}^{1} \mu(\theta(x, \tau))\left[v_{x}^{2}(x, \tau)\right]_{t} d x d \tau=0
$$

whence, after an integration by parts,

$$
\begin{aligned}
\int_{0}^{t} \int_{0}^{1} \sigma_{x}^{2}(x, \tau) d x d \tau+\frac{1}{2} \int_{0}^{1} \mu(\theta(x, t)) v_{x}^{2}(x, t) d x \\
\quad-\frac{1}{2} \int_{0}^{t} \int_{0}^{1} \mu^{\prime}(\theta(x, \tau)) \mu(\theta(x, \tau)) v_{x}^{4}(x, \tau) d x d \tau \\
=\frac{1}{2} \int_{0}^{1} \mu\left(\theta_{0}(x)\right) v_{0 x}^{2}(x) d x .
\end{aligned}
$$

Hence, (2.1) follows from (2.6) by account of (1.2) and (1.3).

In view of (1.7), (2.2) follows immediagely from (2.1) and Schwarz's Inequality.

To show (2.3), we use (2.6) together with

$$
\begin{aligned}
\int_{0}^{1} \sigma^{2}(x, t) d x & \leqslant \max _{x \in[0,1]} \mu(\theta(x, t)) v_{x}^{2}(x, t) d x \\
& \leqslant \max _{x \in[0,1]} \mu\left(\theta_{0}(x)\right) \int_{0}^{1} \mu(\theta(x, t)) v_{x}^{2}(x, t) d x .
\end{aligned}
$$

\section{LEMMA 2.2 .}

$$
\theta_{0}(x) \leqslant \theta(x, t) \leqslant K \text {. }
$$

Proof. We multiply (1.6) by $\mu(\theta)$ and use (1.1) and (2.2) to deduce

$$
\int_{\theta_{0}(x)}^{\theta(x, t)} \mu(\xi) d \xi=\int_{0}^{t} \sigma^{2}(x, \tau) d \tau \leqslant K
$$

Hence, (2.8) and (1.4) imply (2.7).

We now proceed to estimate the $L^{2}$ norm of $\sigma_{x}(x, t)$. 


\section{LEMMA 2.3.}

$$
\int_{0}^{1} \sigma_{x}^{2}(x, t) d x \leqslant K e^{-K t} .
$$

Proof. Using (1.5), (1.7), (1.6) and (1.1),

$$
\begin{aligned}
\frac{1}{2} \frac{d}{d t} & \int_{0}^{1} \sigma^{2}(x, t) d x \\
& =\int_{0}^{1} \sigma_{x}(x, t) \sigma_{x t}(x, t) d x \\
& =\int_{0}^{1}\left[v_{t}(x, t)-f(t)\right] \sigma_{x t}(x, t) d x=\int_{0}^{1} v_{t}(x, t) \sigma_{x t}(x, t) d x \\
& =-\int_{0}^{1} \sigma_{t}(x, t) v_{t x}(x, t) d x \\
& =-\int_{0}^{1} \mu(\theta(x, t)) v_{x t}^{2}(x, t) d x \\
& -\int_{0}^{1} \mu^{\prime}(\theta(x, t)) \mu(\theta(x, t)) v_{x}^{3}(x, t) v_{x t}(x, t) d x
\end{aligned}
$$

or, by virtue of (1.5) and (1.1).

$$
\begin{aligned}
& \frac{d}{d t} \int_{0}^{1} \sigma_{x}^{2}(x, t) d x+2 \int_{0}^{1} \mu(\theta(x, t)) \sigma_{x x}^{2}(x, t) d x \\
& \quad=-2 \int_{0}^{1} \mu^{\prime}(\theta(x, t)) \mu(\theta(x, t)) v_{x}^{3}(x, t) v_{t x}(x, t) d x
\end{aligned}
$$

Because of (2.7), $\left|\mu^{\prime}(\theta(x, t))\right| \leqslant K, \mu(\theta(x, t)) \geqslant K_{1}>0,0 \leqslant x \leqslant 1$, and hence (1.1), (1.5), (1.2), (1.3), (1.4), (2.7), (2.11) and the Cauchy Inequality imply

$$
\frac{d}{d t} \int_{0}^{1} \sigma_{x}^{2}(x, t) d x+K_{1} \int_{0}^{1} \sigma_{x x}^{2}(x, t) d x \leqslant K_{2} \int_{0}^{1} \sigma^{6}(x, t) d x .
$$

On account of (2.3),

$$
\begin{gathered}
\int_{0}^{1} \sigma^{6}(x, t) d x \leqslant \max _{x \in[0,1]} \sigma^{4}(x, t) \int_{0}^{1} \sigma^{2}(x, t) d x \\
\leqslant K\left(\max _{x \in[0,1]} \sigma^{2}(x, t)\right)^{2} \\
\leqslant K\left\{\int_{0}^{1} \sigma^{2}(x, t) d x \int_{0}^{1} \sigma_{x}^{2}(x, t) d x\right\}
\end{gathered}
$$

hence

$$
\int_{0}^{1} \sigma^{6}(x, t) d x \leqslant K \int_{0}^{1} \sigma_{x}^{2}(x, t) d x .
$$

Combining (2.12) and (2.13), we have

$$
\frac{d}{d t} \int_{0}^{1} \sigma_{x}^{2}(x, t) d x \leqslant K \int_{0}^{1} \sigma_{x}^{2}(x, t) d x .
$$


Integrating (2.14) over $[0, t]$ and using (2.1), we deduce

$$
\int_{0}^{1} \sigma_{x}^{2}(x, t) d x \leqslant K \text {. }
$$

Now, combining (2.12) with

$$
\sigma^{2}(x, t) \leqslant \int_{0}^{1} \sigma_{x}^{2}(x, t) d x
$$

and

$$
\sigma_{x}^{2}(x, t) \leqslant \int_{0}^{1} \sigma_{x x}^{2}(x, t) d x
$$

and putting

$$
\psi(t) \stackrel{\text { def }}{=} \int_{0}^{1} \sigma_{x}^{2}(x, t) d x
$$

we obtain the integrodifferential inequality

$$
\frac{d \psi(t)}{d t}+K_{1} \psi(t) \leqslant K_{2} \psi^{3}(t)
$$

where, by account of (2.1) and (2.15),

$$
\int_{0}^{t} \psi(\tau) d \tau \leqslant K_{0}
$$

and

$$
\psi(t) \leqslant K .
$$

By (2.18) and (2.19), we can find a $t_{0} \in\left[0,2 \mathrm{~K}_{0} \sqrt{K_{2} / K_{1}}\right]$ such that

$$
\psi\left(t_{0}\right)<\frac{1}{2} \sqrt{K_{1} / K_{2}} \text {. }
$$

Therefore, for $t \geqslant t_{0}$,

$$
d \psi(t) / d t \leqslant-\frac{1}{2} K_{1} \psi(t) .
$$

Hence

$$
\psi(t) \leqslant \psi\left(t_{0}\right) e^{-K_{1}\left(t-t_{0}\right) / 2}, \quad \text { for } t \geqslant t_{0} .
$$

Combining this with (2.19), we obtain (2.9) for $t \geqslant 0$.

On account of (2.9), (1.7),

$$
\sigma^{2}(x, t) \leqslant K e^{-K t},
$$

which proves (1.14).

Using (1.1), (1.2), (1.3), (1.4), (2.20) and (2.7).

$$
v_{x}^{2}(x, t) \leqslant K e^{-K t} .
$$

Hence, as $t \rightarrow \infty$,

$$
v(x, t)=v_{\infty}(t)+O\left(e^{-K t}\right) .
$$

Integrating (1.5) over $x=[0,1]$ and using (1.7) and (2.22), we arrive at (1.13). 
Combining (1.1) with (1.6),

$$
\sigma^{2}(x, t)=\mu(\theta(x, t)) \theta_{t}(x, t) .
$$

Differentiating (2.23) with respect to $x$, integrating over $[0, t]$ and using $\mu_{x}(\theta(x, t)) \theta_{t}(x, t)$ $=\mu_{t}(\theta(x, t)) \theta_{x}(x, t)$, we obtain

$$
\mu(\theta(x, t)) \theta_{x}(x, t)=\mu\left(\theta_{0}(x)\right) \theta_{0 x}(x)+2 \int_{0}^{t} \sigma(x, \tau) \sigma_{x}(x, \tau) d \tau .
$$

Integrating (2.12) over $[0, t]$ and using $(2.20)$ we find

$$
\int_{0}^{t} \int_{0}^{1} \sigma_{x x}^{2}(x, \tau) d x d \tau \leqslant K
$$

Using (2.25), (2.20), (2.9), (2.24),

$$
\sigma_{x}^{2}(x, t)=\int_{0}^{1} \sigma_{x}^{2}(x, t) d x+2 \int_{0}^{1} \int_{y}^{x} \sigma_{x}(\xi, t) \sigma_{x x}(\xi, t) d \xi d y,
$$

and Schwarz's Inequality, we obtain (1.16).

Since, for every $0 \leqslant x \leqslant 1, \theta(x, t)$ is an increasing function of $t, \theta(x, t)$ converges as $t \rightarrow \infty$, to $\theta_{1}(x)<\infty$. Furthermore, by (1.16) and the Arzela-Ascoli theorem the convergence is uniform in $x$. This proves (1.15). Once the a-priori estimates have been established, the existence of solutions can be established by standard techniques so we will not give the details here.

\section{REFERENCES}

[1] C. M. Dafermos and L. Hsiao, Adiabatic shearing of incompressible fluids with temperature-dependent viscosity, Quart. Appl. Math. Vol. 41 (1983) 